\title{
Cristallisation du silicium amorphe par laser à excimères
}

\author{
D. Pribat, P. Legagneux, F. Plais, F. Petinot, O. Huet et C. Reita \\ THOMSON CSF, Laboratoire Central de Recherche, Domaine de Corbeville, \\ 91404 Orsay cedex, France
}

\begin{abstract}
Résumé : Dans le contexte des écrans plats à cristal liquide, on présente dans cet article une analyse détaillée du mécanisme de cristallisation du silicium amorphe par laser à excimères. On montre en particulier que pour certaines conditions d'irradiation, les couches de silicium polycristallin obtenues permettent de fabriquer des transistors MOS présentant des mobilités $n$ et $p$ (respectivement de 350 et $150 \mathrm{~cm}^{2} / \mathrm{Vs}$ ) comparables à celles obtenues avec du matériau monocristallin sur isolant.
\end{abstract}

\section{INTRODUCTION}

Les matériaux semi-conducteurs en couches minces suscitent beaucoup d'intérêt dans les domaines de la visualisation (écrans plats à cristaux liquides), des capteurs d'images (cassettes $X$ pour applications en radiographie) et de la conversion photovoltaíque. Parmi ces différents matériaux, le silicium polycristallin est probablement le plus attractif pour plusieurs raisons. Tout d'abord, il est monoélément (comparé par exemple à $\mathrm{CdTe}$ ou même au silicium amorphe hydrogéné) ce qui simplifie sa mise en oeuvre et son obtention de manière reproductible. En second lieu, il possède des propriétés de transport qui pour certaines conditions de préparation, se rapprochent de celles du silicium monocristallin. Il est de plus intrinsèquement stable, à la différence par exemple du silicium amorphe hydrogéné. Enfin, il permet la réalisation de transistors de types $n$ et $p$ présentant des caractéristiques équilibrées, ce qui ouvre la possibilité de fabriquer des circuits CMOS.

Le silicium polycristallin est principalement étudié dans le domaine des écrans plats à cristal liquide à matrice active, où son utilisation permet d'intégrer à la périphérie des plaques de verre les circuits d'échantillonage du signal vidéo et d'adressage de l'écran. Ceci représente un intérêt non seulement économique, mais aussi technique, en particulier dans le cas de la projection d'images animées (à cadence vidéo) à base d'écrans à cristaux liquides. En effet, on utilise pour cette application des écrans de petites tailles (afin de minimiser les coûts du système optique de projection) et le report des circuits monolithiques d'adressage à la périphérie de la plaque de verre devient problématique, le pas de connexion étant faible, typiquement inférieur à $50 \mu \mathrm{m}$.

Après une brève présentation du principe de fonctionnement des écrans à cristaux liquides et des écrans à matrice active, l'essentiel de cet article sera consacré aux méthodes d'obtention du silicium polycristallin et particulièrement à la cristallisation par faisceau laser d'un précurseur amorphe (a-Si). Cette technique est en effet très 
prometteuse, car elle permet d'obtenir des films polycristallins pratiquement exempts de défauts intragranulaires.

\section{LES ECRANS A CRISTAUX LIQUIDES ET LES MATRICES ACTIVES [1]}

De façon schématiqué, une cellule à cristal liquide est constituée de deux polariseurs croisés séparés par une lame mince de cristal liquide. Les polariseurs sont montés sur deux plaques de verre typiquement espacées de quelques microns, entre lesquelles est inséré le matériau cristal liquide.

Ce dernier module la transmission de la lumière, sous l'action d'un champ électrique que l'on applique entre les deux plaques de verre par l'intermédiaire de deux électrodes transparentes (constituées d'un film mince d'oxide d'étain et d'indium). La figure 1 présente le principe opératoire d'une cellule utilisant un cristal liquide nématique en hélice.

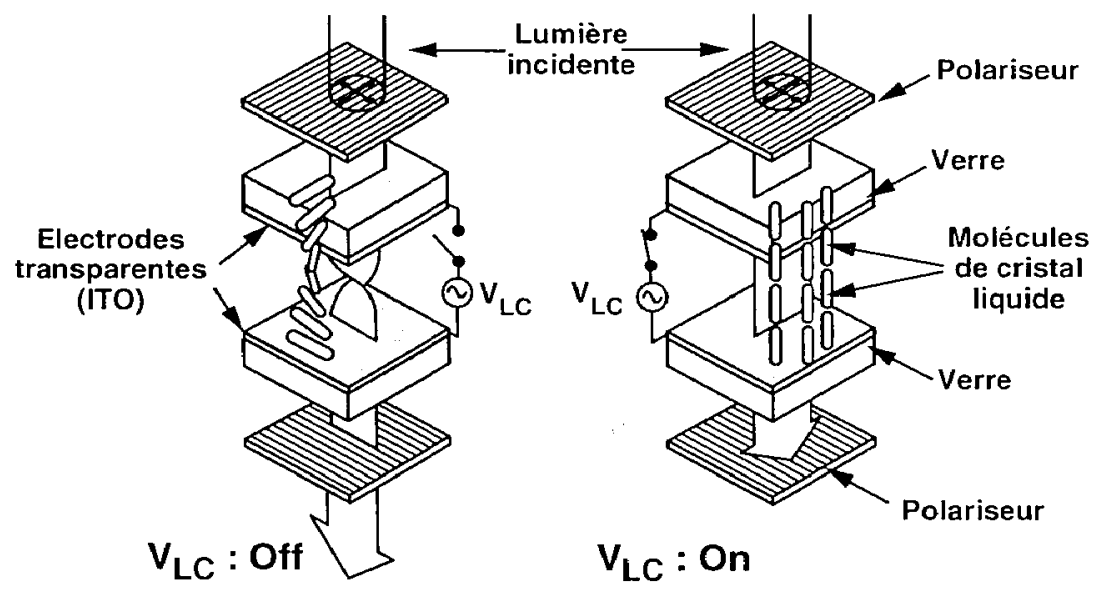

(a)

(b)

Fig. 1 : Structure schématique et principe de fonctionnement d'une cellule a cristal liquide nématique twisté. La lumière est transmise lorsque la cellule n'est pas sous tension (a) alors que la transmission est bloquée lorsque la cellule est polarisée sous quelques volts (b).

Dans une telle cellule, les molécules de cristal liquide sont préalablement alignées, de sorte que leur grand axe soit parallèle à l'axe du polariseur sur chaque plaque de verre. On utilise pour cet alignement des films de polyimide que l'on brosse dans la direction appropriée. Le sens de brossage des films de polyimide étant croisé, les molécules de cristal liquide décrivent entre les deux plaques de verre une hélice dont le pas est égal à 4 fois l'espace interplaque. En l'absence de champ électrique entre les 
deux électrodes transparentes, le plan de polarisation de la lumière incidente sur la cellule subit, après passage du premier polariseur, une rotation progressive de $90^{\circ}$ au cours de la traversée de la lame de cristal liquide et la lumière est transmise (fig. 1a). Par contre, si l'on applique un champ électrique entre les plaques de verre (fig.1b), les molécules de cristal liquide vont avoir tendance à s'aligner avec les lignes de champ, le plan de polarisation de la lumière ne sera plus dévié et le second polariseur (l'analyseur) bloquera la transmission. Le passage de l'état "on" à l'état "off" s'effectue progressivement en fonction de la tension appliquée, ce qui permet d'obtenir des demi teintes ou teintes de gris. On notera que pour obtenir une cellule en couleur, il suffit d'interposer sur le trajet lumineux, un filtre adapté, rouge, vert ou bleu.

Un écran plat à cristal liquide est constitué d'un arrangement plan (bidimensionnel) de cellules (généralement de petites dimensions) telles que celle de la fig. 1, qui sont alors appelées pixel (contraction de picture element). Dans sa configuration la plus simple, l'écran est organisé de façon matricielle, en $\mathrm{n}$ lignes et $\mathrm{m}$ colonnes et l'adressage s'effectue par balayage des $n$ lignes sur un temps de trame. Pendant le temps de sélection de la ligne $i$, les tensions appropriées sont appliquées en parallèle sur les $\mathrm{m}$ colonnes; on passe ensuite à la ligne $\mathrm{i}+1$, etc.., jusqu'à avoir balayé l'ensemble des $\mathrm{n}$ lignes. Le problème de ce type d'adressage réside dans l'apparition de tensions parasites sur les pixels non sélectionnés (par couplage capacitif), ce qui diminue le contraste au fur et à mesure que le nombre de lignes croît (critère de Alt et Pleshko)[2]. De plus, le temps de basculement du cristal liquide (de une à quelques dizaines de $\mathrm{ms}$ ) ainsi que le temps de retour à l'équilibre, imposent des restrictions sévères sur la vitesse de balayage de l'écran, rendant ce mode d'adressage (mutiplexage) incompatible avec l'affichage d'images vidéo de qualité.

Les écrans à matrice active (AMLCDs) ont été developpés afin de pallier les inconvénients énoncés ci-dessus. Le principe est d'introduire au niveau de chaque pixel et en série avec la capacité du cristal liquide un élément de contrôle non linéaire, qui permet de maintenir localement la tension nécessaire au basculement du cristal liquide lorsque la ligne correspondante est désactivée. $L$ 'adressage de l'écran est ainsi découplé du phénomène de basculement du cristal liquide. La figure $2 a$ montre le schéma électrique tandis que la figure $2 b$ représente la structure schématique de ce type d'écran, utilisant des transistors en couches minces (TFTs) comme éléments de contrôle non linéaires.

A l'heure actuelle, les écrans à matrice active sont majoritairement fabriqués avec des TFTs de pixel en silicium amorphe hydrogéné ( $\mathrm{a}-\mathrm{Si}: \mathrm{H}$ ) [3] et l'électronique d'échantillonnage et d'adressage en Si monocristallin est rapportée à la périphérie de la dalle de verre avec des rendements de montage d'autant meilleurs que les pas de répétition entres les lignes et entre les colonnes de la matrice sont importants (cas typique des écrans à vision directe). Pour les applications type projection vidéo, qui utilisent des écrans de petites tailles à forte résolution (i.e. à grand nombre de lignes et colonnes), le report des circuits périphériques devient hasardeux et leur intégration devient pratiquement incontournable. Le silicium polycristallin (Si-poly) a été introduit car les propriétés de transport de a-Si:H (mobilité de l'ordre de $1 \mathrm{~cm}^{2} / \mathrm{Vs}$ pour les électrons et $10^{-3} \mathrm{~cm}^{2} / \mathrm{Vs}$ pour les trous) ne permettent pas de réaliser les registres à décalage et autres fonctions nécessaires à l'adressage des pixels, sauf à utiliser un grand nombre d'entrées vidéo en parallèle [4]. Le Si-poly a tout d'abord été processé à haute température $\left(\sim 1000^{\circ} \mathrm{C}\right)$, sur des substrats de quartz, en utilisant des procédés ainsi que 
des chaînes de production CMOS. Cependant, le coût élevé des substrats de quartz limitant fortement les applications, les équipes de R\&D ont rapidement cherché à développer des procédés basse température, compatibles avec les substrats en verre traditionnellement utilisés dans le domaine des écrans à cristaux liquides.

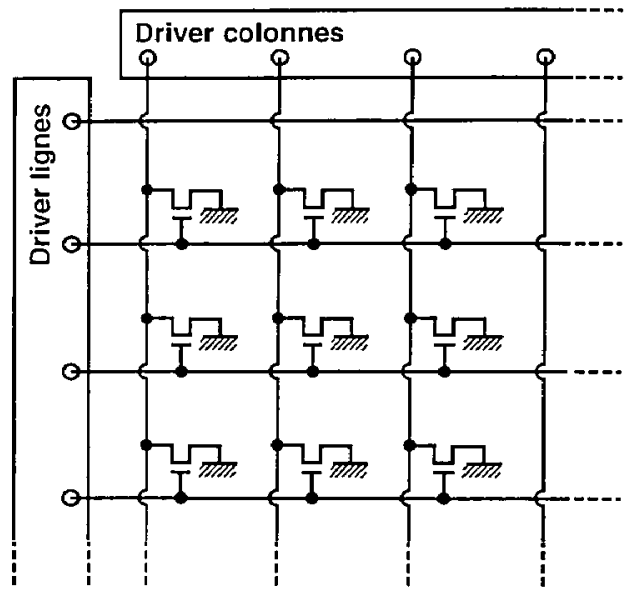

(a)

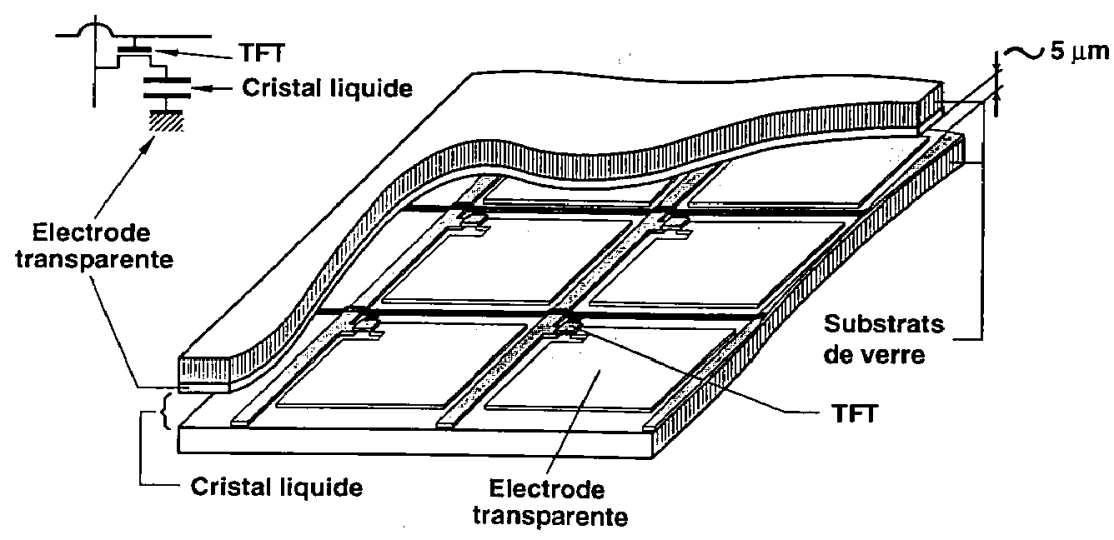

(b)

Fig. 2 : Structure schématique d'un écran à cristal liquide à matrice active. Architecture électrique (a) et vue schématique en perspective (b). 


\section{3- LE SILICIUM POLYCRISTALUIN BASSE TEMPERATURE}

Le Si-poly est généralement obtenu par cristallisation d'un précurseur amorphe. En effet, bien qu'il soit possible d'obtenir du matériau polycristallin directement par dépôt chimique en phase vapeur, (CVD) autour de $600^{\circ} \mathrm{C}$ [3], ce dernier présente en général un état de surface rugueux qui se prête mal au transport électronique de surface (cas typique des composants à effet de champ), les aspérités superficielles diffusant les porteurs, dégradant ainsi leurs mobilités. Les conditions de dépôt conduisant à l'obtention d'un "bon" amorphe ont donc été soigneusement étudiées et rapportées dans la littérature $[3,5]$.

Les conditions de dépôt du précurseur amorphe étant déterminées, les études ont tout d'abord porté sur la cristallisation en phase solide de ce matériau. Typiquement, cette opération est effectuée à $600^{\circ} \mathrm{C}$, pendant des durées de quelques dizaines d'heures et l'on peut obtenir des tailles de grains allant jusqu'à $5 \mu \mathrm{m}$ (pour des épaisseurs de couches de $100 \mathrm{~nm}$ ) lorsque les conditions de dépôt du précurseur amorphe sont optimisées [3,5]. La figure 3 présente une vue au microscope électronique en transmission (MET) de ce type de couche. Il apparaît clairement que la densité de défauts étendus est très importante, avec en particulier un maclage systématique des grains. Ce maclage est inhérent au mode de cristallisation en phase solide, car il est initié aussi bien au cours de la nucléation des grains (étape de formation des embryons critiques) qu'au cours de leur croissance [5]. Il est clair que ces différents défauts étendus vont notablement dégrader le transport électronique dans le matériau et que les TFTs correspondants seront de qualité médiocre par rapport à leurs homologues en Si monocristallin.

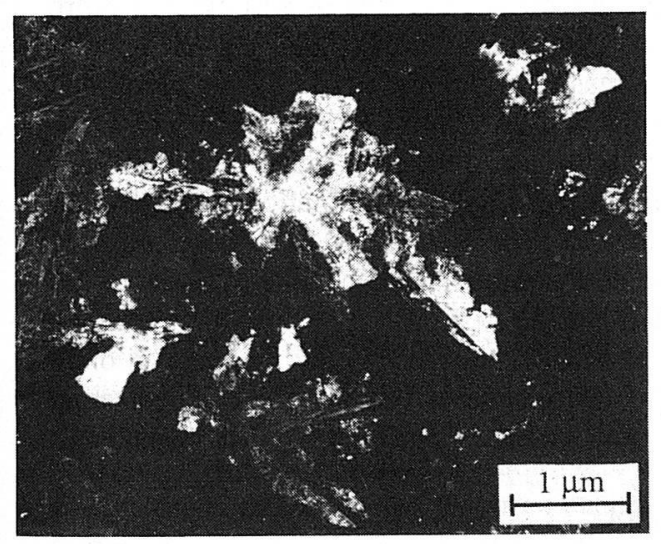

Fig. 3 : Vue plane au microscope électronique en transmission d'une couche de Si-poly cristallisé en phase solide à $580^{\circ} \mathrm{C}$. On notera la grande densité de défauts intragranulaires.

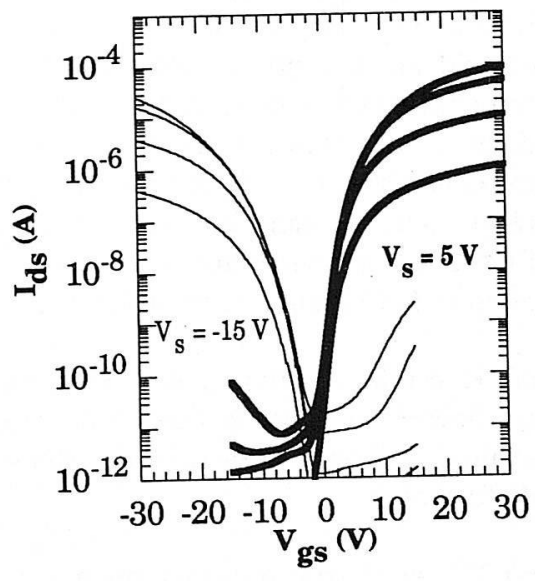

Fig. 4 : Caractéristiques de transfert de transistors réalisés à partir de silicium cristallisé en phase solide à $580^{\circ} \mathrm{C}(\mathrm{W} / \mathrm{L}=3 \mu \mathrm{m} / 10 \mu \mathrm{m}-\mathrm{Vds}=0.1$, 1,5 et $10 \mathrm{~V}$ ). Les mobilités $n$ et $p$ sont respectivement de 60 et $40 \mathrm{~cm}^{2} / \mathrm{Vs}$. 
La figure 4 présente des caractéristiques de transfert typiques de ces TFTs. Ces derniers sont réalisés à l'aide d'un oxyde de grille $\left(\mathrm{SiO}_{2}\right)$ déposé à basse température $\left(\sim 100^{\circ} \mathrm{C}\right)$ en plasma microondes [6] tandis que les zones de source et de drain sont dopées par implantation ionique ( $\mathrm{P}$ ou $\mathrm{B}$ selon le type). Les mobilités $\mathrm{n}$ et $\mathrm{p}$ sont respectivement de 60 et $40 \mathrm{~cm}^{2} / \mathrm{Vs}$ à comparer à $\sim 550$ et $\sim 300 \mathrm{~cm}^{2} / \mathrm{Vs}$ pour du silicium monocristallin sur isolant [7]. De plus, les tensions de seuil sont élevées, (5 et $-15 \mathrm{~V}$ ), ce qui pose un problème pour la fabrication de circuits logiques performants. Ainsi que montré ci-après, la cristallisation par faisceau laser permet de notablement améliorer les performances de ces TFTs.

\section{4- LA CRISTALLISATION DU a-Si PAR FAISCEAU LASER PULSE}

Le traitement des matériaux semiconducteurs par laser pulsé a été initié vers le milieu des années 70 par des chercheurs soviétiques [8]. Les premiers travaux ont porté sur l'activation des dopants et la guérison des défauts dans le silicium monocristallin après implantation ionique.

Dans les matériaux semiconducteurs, l'absorption de photons d'énergie supérieure au gap s'effectue essentiellement par création de paires électrons-trous. Le système de porteurs photogénérés s'équilibre rapidement $\left(\sim 10^{-14} \mathrm{~s}\right)$ et l'énergie est transmise au réseau cristallin par émission de phonons sur un temps de l'ordre de $10^{-12} \mathrm{~s}$, provoquant son échauffement [9]. $L$ 'intérêt des lasers pulsés réside dans le fait que l'énergie reste localisée dans une épaisseur de matériau très faible, ce qui permet d'obtenir la fusion superficielle, sans que le substrat sous-jacent soit thermiquement affecté [10]. Si l'on considère par exemple le cas des lasers $\mathrm{UV}$, la profondeur d'absorption, $\alpha^{-1}$ dans le $\mathrm{Si}$ est de l'ordre de $5 \mathrm{~nm}$ et elle varie peu selon que le matériau est amorphe, cristallin ou liquide. D'autre part, la longueur de diffusion thermique, $l_{t h}$ dans le a-Si est de l'ordre de $150 \mathrm{~nm}$ à haute température $\left(l_{\text {th }} \sim(\mathrm{D} \tau)^{1 / 2}\right.$, avec $\mathrm{D}=\kappa / \rho \mathrm{C}_{\mathrm{p}}$, où $\kappa, \rho$ et $\mathrm{C}_{\mathrm{p}}$ sont respectivement la conductivité thermique, la masse volumique et la chaleur spécifique du a-Si, voir table 1 , et avec $\tau$ durée de l'impulsion laser, prise ici égale à $50 \mathrm{~ns}$ ). Dans ces conditions, $\alpha^{-1}<l_{t h}$ et l'énergie apportée par le laser pendant la durée de l'impulsion reste confinée dans une épaisseur de l'ordre de $l_{t h}$. Une estimation grossière de l'élévation de température (avant fusion) dans cette épaisseur $l_{t h}$ est alors donnée par la relation $\{1\}$ ci-après, tirée de la conservation de l'énergie dans la couche irradiée:

$$
\Delta \mathrm{T} \sim(1-\mathrm{R}) \mathrm{E} / \rho \mathrm{C}_{\mathrm{p}} l_{t h} \quad\{1\},
$$

où $\mathrm{R}$ est le coefficient de réflection de la surface du a-Si à la longueur d'onde considérée, et $\mathrm{E}$ est la densité d'énergie du faisceau laser (en $\mathrm{J} / \mathrm{cm}^{2}$ ). On peut ainsi évaluer la densité d'énergie, $\mathrm{E}_{\mathrm{s}}$, nécessaire pour amener l'épaisseur $l_{t h}$ de a-Si au seuil de fusion:

$$
\mathrm{E}_{\mathrm{s}} \sim\left(\mathrm{T}_{\mathrm{a}}-\mathrm{T}_{\mathrm{amb}}\right) \rho \mathrm{C}_{\mathrm{p}} l_{\text {th }} /(1-\mathrm{R}) \quad\{2\},
$$

où $T_{a m b}$ et $T_{a}$ sont respectivement la température ambiante et la température de fusion du a-Si. De même, pour fondre totalement l'épaisseur $l_{t h} \mathrm{de} \mathrm{a-Si}$, il faudra apporter une énergie laser de l'ordre de:

où $\mathrm{L}_{\mathrm{a}}$ représente la chaleur latente de fusion du a-Si.

$$
\mathrm{E} \sim \mathrm{E}_{\mathrm{s}}+\rho \mathrm{L}_{\mathrm{a}} l_{t h} /(1-\mathrm{R}) \quad\{3\},
$$

Des modèles détaillés, basés sur la résolution numérique de l'équation de diffusion de la chaleur à une dimension ont été établis, qui permettent de calculer précisément la 
profondeur fondue en fonction de l'énergie laser, ainsi que par exemple, l'évolution de la température en fonction du temps à une profondeur donnée $[11,12]$.

Table 1: Données optiques et thermiques relatives au silicium amorphe (a-Si), au silicium cristallin (c-Si), et au silicium liquide (1-Si). $[11,12]$

\begin{tabular}{|c|c|c|c|c|c|c|}
\hline & $\begin{array}{c}\mathbf{R} \\
\text { (à 308 nm et } \\
\text { au voisinage } \\
\left.\text { de } T_{f}\right)\end{array}$ & $T_{f}(\mathrm{~K})$ & $\rho\left(\mathrm{g} / \mathrm{cm}^{3}\right)$ & $\begin{array}{c}\mathrm{C}_{\mathrm{p}}(\mathrm{J} / \mathrm{g} . \mathrm{K}) \\
\text { (au voisinage } \\
\text { de } \mathrm{T}_{\mathrm{f}} \text { ) }\end{array}$ & $\begin{array}{c}\mathrm{\kappa}(\mathrm{W} / \mathrm{cm} . \mathrm{K}) \\
\text { (ali voisinage } \\
\left.\text { de } \mathrm{T}_{f}\right)\end{array}$ & $\mathrm{L}(\mathrm{J} / \mathrm{g})$ \\
\hline $\mathrm{a}-\mathrm{Si}$ & 0.55 & $\sim 1430$ & 2.26 & 1.1 & 0.015 & $\sim 1280$ \\
\hline $\mathrm{c}-\mathrm{Si}$ & 0.66 & 1687 & 2.31 & 1.0 & 0.23 & 1800 \\
\hline $\mathrm{l-Si}$ & 0.69 & - & 2.52 & 1.0 & 0.6 & - \\
\hline
\end{tabular}

Bien que les premiers travaux de recuit aient été effectués avec des lasers déclenchés solides (lasers YAG ou rubis), la tendance actuelle est à l'utilisation de lasers à gaz et plus particulièrement de lasers à excimères. Ces derniers permettent d'obtenir des faisceaux de dimensions plus importantes, car il y a moins de problèmes de dissipation thermique que dans les lasers solides. De plus, les lasers à excimères sont très peu cohérents, ce qui élimine pratiquement les phénomènes d'interférences et de speckle. Les longueurs d'onde utilisables sont 193, 249,308 et $350 \mathrm{~nm}$ respectivement pour les complexes $\mathrm{ArF}, \mathrm{KrF}, \mathrm{XeCl}$ et $\mathrm{XeF}$.

La cristallisation rapide du a-Si présente des spécifités qu"il convient de bien analyser, afin de pouvoir maitriser les conditions opératoires conduisant à l'obtention d'un polycristal à gros grains, dont on sent intuitivement qu'il donnera les meilleurs résultats en terme de transport électronique.

En conditions de chauffage "normales", le a-Si cristallise avant fusion. Cependant, sous l'effet d'une impulsion laser suffisamment brève, l'apport de chaleur est tel que le matériau fond (par une transition de phase du premier ordre) avant d'avoir cristallisé. La température de fusion du a-Si étant inférieure de $\sim 250^{\circ} \mathrm{C}$ à la température d'équilibre solide-liquide (température de fusion du $\mathrm{c}-\mathrm{Si}, \mathrm{T}_{\mathrm{c}}$ ), il s'ensuit que le liquide ainsi formé est très surfondu, ce qui va favoriser la nucléation rapide de cristallites en son sein (dans les conditions d'irradiation utilisées par Lowndes et al., les premières cristallites apparaissent dans le liquide environ 10 ns après le début de la fusion de la surface [13]). La nucléation et la croissance de ces cristallites libère la chaleur latente de changement d'état du cristal $\left(L_{c}\right)$ dans le liquide, ce qui provoque l'échauffement de ce dernier au dessus de la température de fusion du a-Si. Il s'ensuit que le a-Si contigu au liquide est amené à fusion, et que cette nouvelle transformation absorbe la chaleur latente de liquéfaction du matériau amorphe, $L_{a}$, ce qui empêche le liquide de se réchauffer vers $T_{c}$. La fine couche de liquide nouvellement formée sous les cristallites reste donc en surfusion importante, ce qui entraîne la rapide nucléation de nouvelles cristallites etc... Comme $\mathrm{L}_{c}>\mathrm{L}_{\mathbf{a}}$, (voir tableau 1), la fusion de a-Si sur une fine couche (couche liquide enterrée) traversant progressivement toute l'épaisseur du film est autoentretenue, et ce phénomène est appelé cristallisation explosive [13]. Le taux de nucléation étant très élevé dans la fine couche de liquide surfondu, la cristallisation explosive transforme le film de a-Si en un film de $\mathrm{Si}$ polycristallin à très petits grains, 
sur l'épaisseur intéréssée par le phénomène. La vitesse initiale de propagation de la couche liquide enterrée a été mesurée autour de $12-14 \mathrm{~m} / \mathrm{s}[13,14]$.

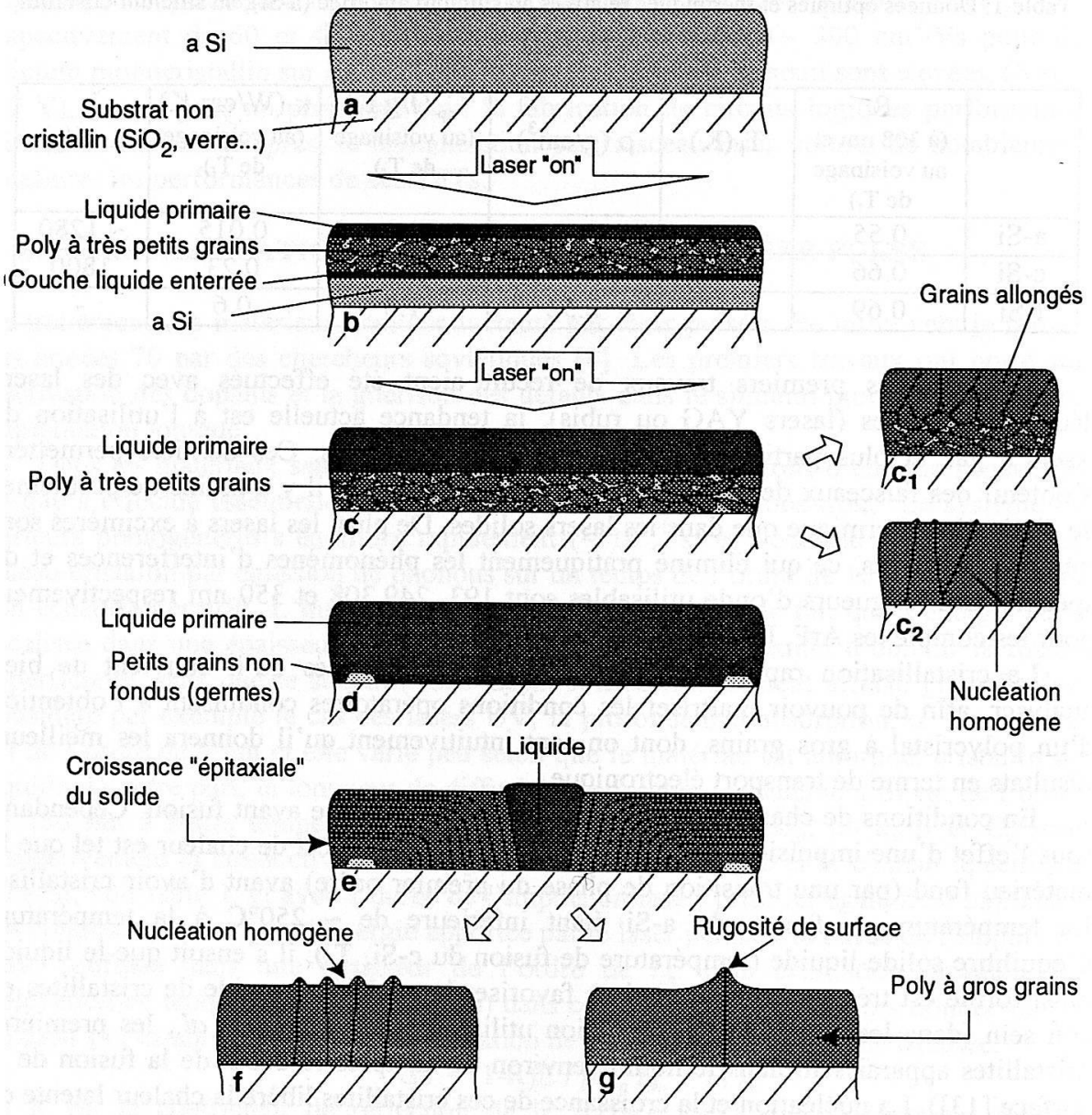

Fig. 5 : Représentation schématique des transfommations de phase ayant lieu au cours de l'irradiation par laser pulsé d'un film mince de a-Si.

A partir des remarques précédentes, la figure 5 montre schématiquement les différentes transformations qui peuvent avoir lieu au cours de l'irradiation d'une couche de a-Si à l'aide d'impulsions laser de quelques dizaines de nanosecondes. La structure de départ est représentée sur la figure $5 \mathrm{a}$. Sur la figure $5 \mathrm{~b}$, on est en cours d'irradiation, une pellicule de liquide primaire (par opposition à liquide enterré) a été formée en surface, ce qui a déclenché la cristallisation explosive; la couche liquide enterrée est représentée au cours de sa progression, laissant derrière elle du polycristal à très petits 
grains. Sur la figure 5c, on est toujours en cours d'irradiation, la couche liquide enterrée a traversé toute l'épaisseur du film de a-Si et ce dernier a été transformé en polycristal à très petits grains; dans le même temps, le liquide primaire a progressé, en fondant le polycristal à très petits grains synthétisé par la cristallisation explosive. Si l'apport énergétique du laser est insuffisant pour permettre la fusion totale du poly à très petit grains, la solidification s'effectue sans nucléation, à partir des cristallites du poly à très petits grains, par "épitaxie" liquide, à la profondeur maximale atteinte par la phase liquide; on obtient un matériau stratifié, avec des grains allongés dans la zone superficielle (fig. $5 \mathrm{c}_{1}$ ). Si le poly à très petits grains est entièrement fondu, la nucléation de la phase solide s'effectue dans le liquide, probablement de manière homogène [15] et l'on obtient un matériau à petits grains, homogène sur l'épaisseur du film (fig. $5 c_{2}$ ). Les figures $5 \mathrm{~d}-\mathrm{g}$ présentent le cas particulier où la densité d'énergie du laser est telle que le poly à très petits grains synthétisé par la cristallisation explosive est pratiquement tout fondu, avec cependant quelques grains épars subsistant à l'interface. Ces petits grains vont servir de germe pour la croissance (non nuclée) de la phase solide (fig. 5e), qui s'effectue latéralement, par consommation du liquide, jusqu'au contact avec la cristallite adjacente. On peut ainsi obtenir des grains de plusieurs microns de diamètre, (fig. $5 \mathrm{~g}$ et photo fig. 6), car la nucléation homogène entre les cristallites en cours de croissance est inhibée par le rejet de la chaleur latente de cristallisation qui réchauffe le liquide intragranulaire autour de $T_{c}$. Par contre, si la distance entre les petits grains (les germes) persisțant à l'interface est trop importante, le liquide intragranulaire n'est plus isotherme et la nucléation homogène se déclenche entre les gros grains en croissance, ce qui conduit à un matériau "biphasé", avec des régions à petits grains, entourées de très gros grains (schéma fig. 5f et photo fig. 6). Dans les deux derniers cas considérés, l'écoulement de la chaleur n'est plus unidimensionnel, ce qui rend la modélisation beaucoup plus complexe [16].

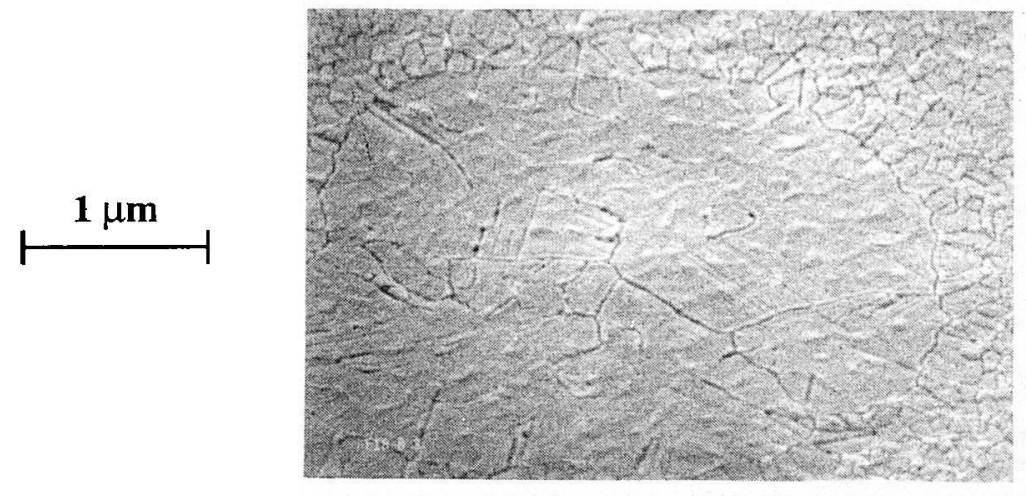

Fig. 6 : Vue au microscope électronique à balayage (après révélation chimique des joints de grain) d'un film mince de Si-poly cristallisé laser a $580 \mathrm{~mJ} / \mathrm{cm}^{2}$. On notera que le grain central (diamètre $\sim 3 \mu \mathrm{m}$ ) est entouré de petits grains, illustrant la figure $5 f$ et son commentaire. 
On a reporté sur la figure 7 les caractéristiques de transfert de TFTs réalisés dans du polycristal à très gros grains. Ces films ont été obtenus par irradiation à l'aide d'un laser $\mathrm{XeCl}(\tau \sim 30 \mathrm{~ns})$ d'un dépôt de a-Si de $80 \mathrm{~nm}$ d'épaisseur. L'irradiation, a été effectuée à $580 \mathrm{~mJ} / \mathrm{cm}^{2}$, avec un recouvrement de faisceau de $90 \%$, tandis que le substrat était maintenu à $350^{\circ} \mathrm{C}$ [17]. Les caractéristiques de ces TFTs sont bien meilleures que celles présentées plus haut (mobilités $\mathrm{n}$ et $\mathrm{p}$ de 350 et $150 \mathrm{~cm}^{2} / \mathrm{Vs}$, tensions de seuil $n$ et $p$ de 1,2 et $-2,4 \mathrm{~V}$ ) et elles deviennent comparables à celles de MOSFETs fabriqués dans du silicium monocristallin sur isolant [7], attestant de la grande qualité des films obtenus par cristallisation laser. On peut apprécier cette qualité cristalline sur la photo de microscopie à transmission présentée figure 8 . Concernant les paramètres des TFTs, des résultats similaires ont été publiés récemment [18-20].

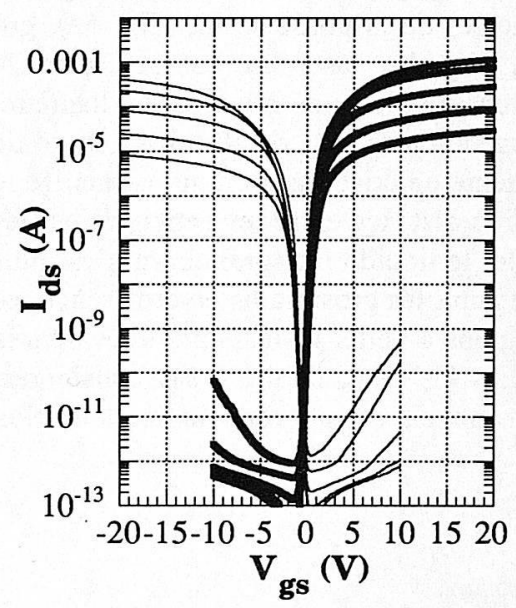

Fig. 7 : Caractéristiques de transfert de transistors réalisés à partir de silicium cristallisé par laser à $580 \mathrm{~mJ} / \mathrm{cm}^{2}(\mathrm{~W} / \mathrm{L}=10 \mu \mathrm{m} / 5 \mu \mathrm{m}$ $\mathrm{Vds}=0.1,0.3,1,3$ et $6 \mathrm{~V}$ ). Les mobilités n et $p$ sont respectivement de 350 et $150 \mathrm{~cm}^{2} / \mathrm{Vs}$.

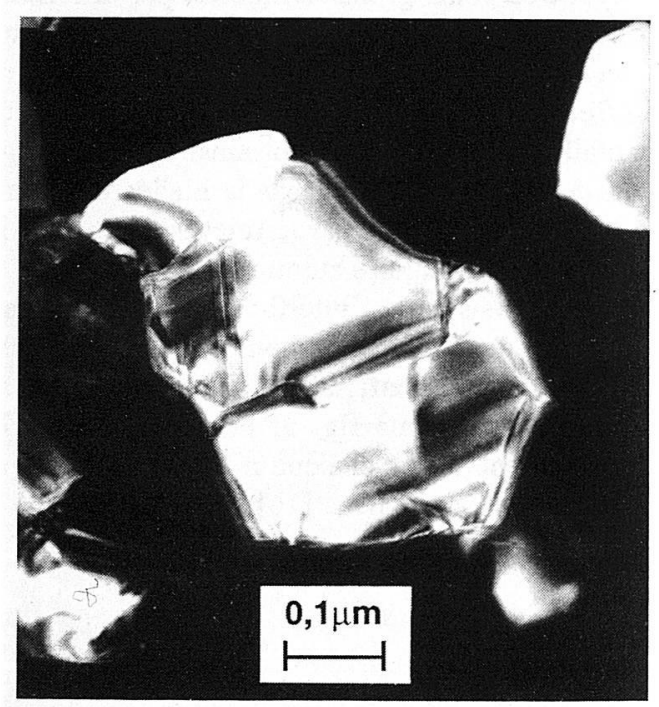

Fig. 8 : Vue plane au microscope électronique en transmission d'un grain de Si-poly cristallisé laser. On notera la faible densité de défauts intragranulaires et la netteté des joints de grains.

Il est clair, au vu du mécanisme décrit sur la figure 5, qu'il faut pouvoir contrôler très précisement la densité d'énergie délivrée par le faisceau laser (tant spatialement que temporellement) si l'on veut obtenir des films polycristallins à très gros grains présentant une structure homogène sur de grandes surfaces. Pour ce faire, on utilise des faisceaux laser soigneusement homogénéisés à l'aide de réseaux de lentilles sphériques (ou cylindriques, couplés dans deux directions perpendiculaires) et l'on irradie plusieurs fois la même surface, de manière à moyenner les variations d'énergie pulse à pulse. De plus, on limite le recouvrement du spot à une seule direction, en donnant au faisceau une forme très allongée. Les systèmes d'homogénéisation actuellement à l'étude 
devraient permettre de délivrer des faisceaux de $250-300 \mathrm{~mm}$ de long sur $\sim 500 \mu \mathrm{m}$ de large, ce qui permettra de traiter des plaques de verre de plus de $50 \mathrm{~cm}$ de large en seulement deux passes.

\section{5- CONCLUSION}

La cristallisation du a-Si par laser excimère apparait extrêmement attractive car elle permet d'obtenir des films de Si-poly de grande qualité, avec un budget thermique réduit. On a montré dans cet article qu'à partir de tels films, on pouvait fabriquer des transistors MOS présentant des mobilités $n$ et $p$ de respectivement 350 et $150 \mathrm{~cm}^{2} / \mathrm{Vs}$, ce qui devient comparable aux valeurs obtenues avec du silicium monocristallin sur isolant. Le procédé n'est pas encore industriel, mais au vu des efforts de développement consentis par les fabricants d'écrans plats, particulièrement au Japon, il est clair que les problèmes d'uniformité en énergie et de reproductibilité pulse à pulse d'une part ainsi que de maintenance d'équipement d'autre part seront rapidement résolus, ouvrant ainsi la voie à de multiples applications.

\section{Références}

[1] voir par ex. : Kaneko E., Liquid crystal displays (KTK Scientific Publishers, Tokyo, 1991)

[2] Alt P. M., Pleshko P., IEEE Trans. Electron Dev., ED-21 (1974) 146; voir aussi Budin J.P., « The basic of flat panel display addressing », Seminar Lecture Notes, The 13th International Display Research Conference, Strasbourg 31 August 1993, (Le Club Visu-SID, 1993) pp. A1/1-A1/38.

[3] Pribat D., Plais F., Legagneux P., Reita C., Science and technology of thin films, F.C. Mattacotta and G. Ottaviani Eds., (World Scientific, Singapore, 1995) pp. 293 359.

[4] Lebrun H., Szydlo N., Maurice F., Borel T., Stewart R.G., Weisbrod S., Huq R., « a-Si self-scanned 2.2-in. EDTV projection LC light valve» Society for Information Display International Symposium, San Diego 12-17 May 1996 (SID, Santa Ana 1996) pp. 677-680.

[5] Pribat D., Legagneux P., Plais F., Reita C., Petinot F., Huet O., « Low temperature polysilicon materials and devices ", Materials Research Society Spring Meeting, Symposium on Flat Panel Display Materials, San Francisco 8-12 April 1996, to be published.

[6] Jiang N., Hugon M.C., Agius B., Kretz T., Plais F., Pribat D., Carrière T., Puech M., Jpn. J. Appl. Phys., 31 (1992) L1014.

[7] Colinge J.P., Silicon-on-insulator technology: Materials to VLSI (Kluwer Academic Publishers, 1991)

[8] Shtyrkov E.I., Khaibullin I.B., Zaripov M.M., Galyatudinov M.F., Bayazitov R.M., Sov. Phys. Semicond., 9 (1976) 1309

[9] Wood R.F., Gellison G. E., Pulsed laser processing of semiconductors, Semiconductors and Semimetals, Vol. 23, R.F. Wood, C.W. White and R.T. Young Eds., (Academic Press 1984) pp. 165-250.

[10] Sameshima T., Hara M., Usui S., Jpn. J. Appl. Phys., 28 (1989) L2131.

[11] Wood R.F., Geist G.A., Phys. Rev., B34 (1986) 2606. 
[12] de Unamuno S., Fogarassy E., Appl. Surf. Science, 36 (1989) 1.

[13] Lowndes D.H., Pennycook S.J., Jellison G.E., Withrow S.P., Mashburn D.N., J. Mater. Res., 2 (1987) 648.

[14] Murakami K., Eryu O., Takita K., Masuda K., Phys. Rev. Lett., 59 (1987) 2203

[15] Stiffler S.R., Thompson M.O., Peercy P.S., Phys. Rev. Lett., 60 (1988) 2519.

[16] Lowndes D.H., Pennycook S.J., Wood R.F., Jellison G.E., Withrow S.P., Mat.

Res. Soc. Symp. Proc., 100 (1988) pp. 489-503.

[17] Legagneux P., Petinot F., Huet O., Plais F., Reita C., Pribat D., Carluccio R., Pecora A., Mariucci L., Fortunato G., « High quality polycrystalline thin film transistors made by excimer laser crystallisation ", European Solid State Device Reseach Conference, Bologna 8-11 September 1996, to be published.

[18] Fogarassy E., Prevot B., de Unamuno S., Elliq M., Pattyn H., Mathe E. L., Naudon A., Appl. Phys., A56 (1993) 365.

[19] Kuriyama H., Nohda T., Aya Y., Kuwahara T., Wakisaka K., Kiyama S. Tsuda S., Jpn. J. Appl. Phys., 33 (1994) 5657.

[20] Sameshima S., Khono A., Sekiya M., Hara M., Sano N., Appl. Phys. Lett. 64 (1994) 1018. 